\title{
CORRECTION
}

\section{Author Correction: Intragenic antagonistic roles of protein and circRNA in tumorigenesis}

\author{
Jlenia Guarnerio ${ }^{1,2}$, Yang Zhang ${ }^{1}$, Giulia Cheloni ${ }^{1}$, Riccardo Panella ${ }^{1}$, Jesse Mae Katon ${ }^{1}$, Mark Simpson ${ }^{3}$, Akinobu Matsumoto ${ }^{1}$, \\ Antonella Papa (iD) ${ }^{1}$, Cristian Loretelli ${ }^{1}$, Andreas Petri ${ }^{1}$, Sakari Kauppinen ${ }^{4}$, Cassandra Garbutt ${ }^{5}$, Gunnlaugur Petur Nielsen ${ }^{6}$, \\ Vikram Deshpande ${ }^{6}$, Mireia Castillo-Martin ${ }^{7}$, Carlos Cordon-Cardo ${ }^{7}$, Dimitrios Spentzos ${ }^{5}$, John G. Clohessy (iD) ${ }^{1}$, Mona Batish ${ }^{3,8}$ and \\ Pier Paolo Pandolfi id ${ }^{9}$
}

Cell Research (2020) 30:188; https://doi.org/10.1038/s41422-019-0262-4

Correction to: Cell Research https://doi.org/10.1038/s41422-0190192-1, published online 17 June 2019

We apologize for author name and affiliation errors that we just identified in our paper published in 2019 August issue. There were mistakes in the name of one author and the location of institutional affiliations for four authors. Specifically, author "Dimitrios Spentzos" was previously listed as "Spentzos Dimitrios" resulting in a reversal of his first and last name. Further, institutional affiliation 5 (for authors Spentzos D. and Garbutt C.): MGH Center for Sarcoma and Connective Tissue Oncology, Department of Orthopedic Surgery, is erroneously listed in "New York, USA". The correct location is "Boston, USA". Similarly, institutional affiliation 6 (for authors Nielsen G.P. and Deshpande V.): MGH Center for Sarcoma and Connective Tissue Oncology, Department of Pathology, is erroneously listed in "New York, USA". The correct location is "Boston, USA". These corrections do not affect the description of the results or the conclusion of this work. We apologize for not detecting them before publication and any inconvenience this caused. The correct author names and institutional affiliation descriptions are as follows:

Jlenia Guarnerio ${ }^{1,2}$, Yang Zhang ${ }^{1}$, Giulia Cheloni ${ }^{1}$, Riccardo Panella ${ }^{1}$, Jesse Mae Katon ${ }^{1}$, Mark Simpson ${ }^{3}$, Akinobu Matsumoto ${ }^{1}$, Antonella Papa ${ }^{1}$, Cristian Loretelli ${ }^{1}$, Andreas Petri ${ }^{1}$, Sakari Kauppinen $^{4}$, Cassandra Garbutt ${ }^{5}$, Gunnlaugur Petur Nielsen ${ }^{6}$, Vikram
Deshpande $^{6}$, Mireia Castillo-Martin ${ }^{7}$, Carlos Cordon-Cardo ${ }^{7}$, Dimitrios Spentzos ${ }^{5}$, John G. Clohessy ${ }^{1}$, Mona Batish ${ }^{3,8}$ \& Pier Paolo Pandolfi ${ }^{9}$

${ }^{1}$ Cancer Research Institute, Beth Israel Deaconess Cancer Center, Departments of Medicine and Pathology, Beth Israel Deaconess Medical Center, Harvard Medical School, Boston, MA, 02215, USA.

${ }^{2}$ Cedars-Sinai Medical Center, Department of Radiation Oncology, Samuel Oschin Comprehensive Cancer Center, Los Angeles, CA, 90048, USA.

${ }^{3}$ Department of Microbiology, Biochemistry and Molecular Genetics, Rutgers University, Newark, NJ, 07103, USA.

${ }^{4}$ Center for RNA Medicine, Department of Clinical Medicine, Aalborg University, Copenhagen, Denmark; Department of Hematology, Aalborg University Hospital, Aalborg, Denmark.

${ }^{5} \mathrm{MGH}$ Center for Sarcoma and Connective Tissue Oncology, Department of Orthopedic Surgery, Boston, USA.

${ }^{6} \mathrm{MGH}$ Center for Sarcoma and Connective Tissue Oncology, Department of Pathology, Boston, USA.

${ }^{7}$ Department of Pathology, Mount Sinai School of Medicine, The Mount Sinai Medical Center, New York, NY, 10029, USA.

${ }^{8}$ Department of Medical and Molecular Sciences, University of Delaware, Newark, DE, 19716, USA.

${ }^{9}$ Cancer Research Institute, Beth Israel Deaconess Cancer Center, Departments of Medicine and Pathology, Beth Israel Deaconess Medical Center, Harvard Medical School, Boston, MA, 02215, USA.

${ }^{1}$ Cancer Research Institute, Beth Israel Deaconess Cancer Center, Departments of Medicine and Pathology, Beth Israel Deaconess Medical Center, Harvard Medical School, Boston,

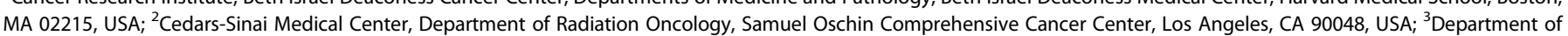

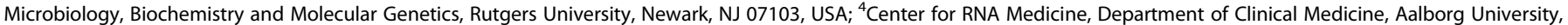

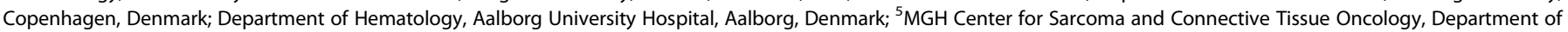

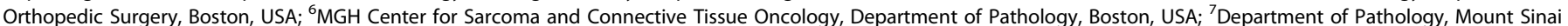

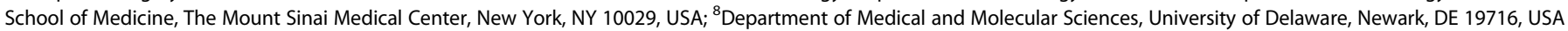

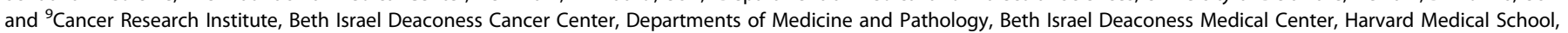
Boston, MA 02215, USA 